\title{
Detection of resonant impurities in graphene by quantum capacitance measurement
}

\author{
Lin Wang, ${ }^{1, *}$ Xiaolong Chen, ${ }^{1, *}$ Wei Zhu, ${ }^{2}$ Yang Wang, ${ }^{1}$ Chao Zhu, ${ }^{1}$ Zefei Wu, ${ }^{1}$ Yu Han, ${ }^{1}$ \\ Mingwei Zhang, ${ }^{1}$ Wei Li, ${ }^{1}$ Yuheng He, ${ }^{1}$ and Ning Wang ${ }^{1, \dagger}$ \\ ${ }^{1}$ Department of Physics and the William Mong Institute of Nano Science and Technology, The Hong Kong University of Science and \\ Technology, Hong Kong, China \\ ${ }^{2}$ Department of Physics and Astronomy, California State University, Northridge, California 91330, USA \\ (Received 7 January 2014; revised manuscript received 22 January 2014; published 10 February 2014)
}

\begin{abstract}
We investigated Ag-adatom-induced resonant impurities of graphene by quantum capacitance measurement. Different from charged impurities and other conventional resonant impurities, Ag atoms form very weak covalent bonds with graphene. The Ag-adatom-induced resonant peak as measured by quantum capacitance grows more intense at cryogenic temperatures, at higher impurity concentrations, and in stronger magnetic fields, in accordance with our theoretical calculations. The appearance of resonant states and the split of the zeroth Landau level for Ag-adsorbed graphene are manifestations of the formation of a flat impurity band near the Dirac point.
\end{abstract}

DOI: 10.1103/PhysRevB.89.075410

\section{INTRODUCTION}

Disorder has profound impacts on graphene's extraordinary properties and is often intentionally introduced to modify the performance of functional graphene-based devices [1-4]. Theoretical predictions suggest that point defects and adsorbates in single-layer graphene induce midgap states near the Dirac point [5-11]. The local density of states (LDOS) in disordered graphene nanostructures has been investigated theoretically and experimentally [5-10,12-17]. Previous scanning tunneling spectroscopy studies have shown that robust LDOS peaks appear near a single vacancy in graphene or in hydrogenated graphene $[12,13,16]$ These resonant impurities, however, have not been successfully detected in transport measurements because their transport behavior always mimics that of charged impurities at finite densities [9,10,18-24]. Different scattering details are involved, and thus no clear resonant feature can be observed in the conductivity $[8-10,21,22,25]$. Alternative methods for effective measurements of the mesoscopic physical behavior of disordered graphene are required, which are essential for probing these resonant impurities and their effects on the average DOS (ADOS) at a much larger scale.

Vacancies in graphene have been well known as the prototype example of resonant impurities. They cause strong electron localization effects and transitions to insulating states in graphene at cryogenic temperatures [18]. The formation of local magnetic moments around vacancies in graphene has been confirmed theoretically and experimentally $[5,12,26]$. However, the study of graphene adsorbed with noble metal adatoms is rather incomplete. It is still under debate whether these adatoms ionically or covalently bond with carbon atoms of graphene. The changes in the electronic properties of graphene induced by noble-metal adatoms (should be different from the case of vacancies, hydrogenous impurities, and transition metal adatoms) need to be addressed $[5,9,12,14,18,23]$. Recently, we reported that a very high

\footnotetext{
*These authors contributed equally to this work.

†Corresponding author: phwang@ust.hk
}

PACS number(s): 73.22.Pr, 72.80.Vp, 73.20.Hb, 81.05.ue

concentration of $\mathrm{Ag}$ adatoms could create graphene systems in a strongly correlated electron regime in which negative quantum capacitance phenomena occur even in the absence of a magnetic field [27].

In this paper, Ag adatoms (one noble metal) adsorbed in graphene with low concentrations were systematically studied. They are resonant impurities but with a very weak bonding energy with graphene $[6,28,29]$. Quantum capacitance (which is proportional to ADOS) [30-33] measurement is a powerful tool to clearly detect that $\mathrm{Ag}$ adatoms induce obvious resonances near the Dirac point and the intensity of these resonances is mainly sensitive to impurity concentrations, temperatures, and magnetic field strengths. Based on density functional theory and tight-binding model simulations, we show that a robust resonant peak exists near the Dirac point in both LDOS and ADOS of Ag-adsorbed graphene, in line with our experimental observations.

\section{EXPERIMENTAL}

Single-layer graphene samples were mechanically exfoliated from Kish graphite and placed on $\mathrm{Si}$ substrates with a 300-nm-thick $\mathrm{SiO}_{2}$ [1]. After characterization by Raman spectroscopy measurements [34], graphene samples were then subjected to the deposition of $\mathrm{Ag}$ adatoms with de plasma sputtering in high base vacuum conditions $\left(10^{-7}\right.$ torr). The insulating layer $\mathrm{Y}_{2} \mathrm{O}_{3}(6-8 \mathrm{~nm}$ in thickness) was fabricated by depositing a thin layer of yttrium of about $5 \mathrm{~nm}$ in thickness using electron-beam evaporation followed by oxidation in air at $180^{\circ} \mathrm{C}$ for $30 \mathrm{~min}$ [35-37]. Drain/source and topgate electrodes $[\mathrm{Ti}(5 \mathrm{~nm}) / \mathrm{Au}(40 \mathrm{~nm})]$ were defined by conventional electron-beam lithography techniques (Raith e-LiNE Nanoengineering Workstation and AST electron-beam evaporation system) [37-39].

Capacitance measurement was performed in a threeterminal configuration at temperatures ranging from $300 \mathrm{~K}$ down to $2 \mathrm{~K}$ using a HP 4284A LCR meter (sensitivity $\sim 0.1 \mathrm{pF}$ ). An ac excitation signal of $15 \mathrm{mV}$ at $100 \mathrm{kHz}$ was used in order to improve accuracy and reduce noise levels. The excitation frequency of $100 \mathrm{kHz}$ is much smaller compared with the value of the cutoff frequency 
( $f_{c}=1 / 2 \pi R C \cong 10^{6} \mathrm{kHz}$ ) for our graphene devices; therefore, the capacitors were fully charged during measurement. All cables in the measurement circuit were electrically shielded, and the Si substrates were grounded to avoid any parasitic capacitance, which was also confirmed to be negligible using the capacitance bridge technique [40]. Actually, we have measured the residual capacitance of the circuit in the same geometry of the devices without graphene. The value is less than $0.3 \mathrm{fF}$, which is of several orders smaller than the measured total capacitance of our samples.

\section{RESULTS AND DISCUSSION}

The Hamiltonian of tight-binding treatment of resonant impurities can be written as

$$
\begin{aligned}
H & =H_{0}+H_{\mathrm{imp}} \\
& =t \sum_{\langle i, j\rangle} c_{i}^{\dagger} c_{j}+\varepsilon_{d} \sum_{i} d_{i}^{\dagger} d_{i}+V \sum_{i}\left(d_{i}^{\dagger} c_{i}+\text { H.c. }\right),
\end{aligned}
$$

where $c_{i}^{\dagger}\left(c_{j}\right)$ and $d_{i}^{\dagger}\left(d_{j}\right)$ denote the creation/annihilation operators of electrons localized in graphene $\pi$ bands and resonant impurities, respectively, and $t$ is the nearest-neighbor hopping energy. The values of on-site energy $\varepsilon_{d}$ and energy-dependent effective potential $V$ are fixed and determined by the type of resonant impurities $[5,6,8,9]$. As can be seen from $H_{\text {imp }}$, the nature of resonant impurities lies in the hybridization between electrons of graphene $\pi$ bands and electrons of the impurity band. To elucidate the behavior of Ag-adsorbed graphene, we firstly performed density functional theory simulations of graphene adsorbed by one single Ag atom. The results shown in Fig. S4 (see Supplemental Materials; Ref. [41]) clearly reveal the appearance of a flat impurity band near the Fermi energy $E_{F}=0[6,7,42,43]$. These results confirm Ag adatoms are resonant impurities (not long-range charged impurities such as $\mathrm{K}$ adatoms) producing Dirac resonances [7,8,28,29]. The best fitting of density functional theory integrating the tight-binding model yields $\varepsilon_{d}=0$ and $V=0.08 t$ [41]. The reason why the impurity-related level $\varepsilon_{d}$ occurs at $E_{F}=0$ is that the level repulsion due to the adsorbate-host coupling always drives $\varepsilon_{d}$ towards the lowest DOS. Ag adatoms bind covalently to carbon atoms of graphene, but the bond strength is very weak compared with that of other types of resonant impurities such as hydrogen adatoms [5,8,16,23,44,45].

To explicitly show the influence of resonant scatters on the electronic structure of graphene, we develop a first-principles method to calculate the effective energy dispersion of graphene in the presence of randomly distributed $\mathrm{Ag}$ adatoms with impurity concentration $n_{i}=0.25 \%, 0.50 \%$, and $0.75 \%$. As shown in Fig. 1(a), the resonance dispersion induced by $\mathrm{Ag}$ impurities is formed near the Dirac point. With increasing impurity concentration, the flat bands are more obvious, and the influenced energy region of the electronic structure of graphene becomes larger [44]. In this framework, both LDOS and ADOS of Ag-adsorbed graphene are calculated based on the tight-binding model as shown in Figs. 1(b)-1(d). Figure 1(b) shows the real space mapping of LDOS in graphene with $\mathrm{Ag}$ adatoms $\left(n_{i}=0.30 \%\right)$ at $E_{F}=0$. The intensities of LDOS close to Ag adatoms are high compared with those in
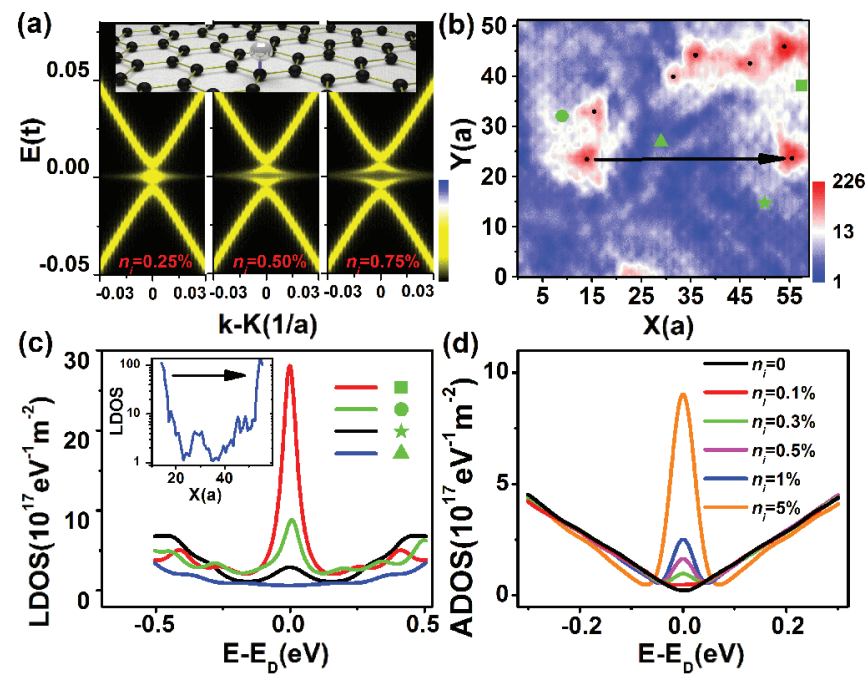

FIG. 1. (Color online) Theoretical calculations for Ag-adsorbed graphene. (a) Effective energy-momentum dispersion relation in the presence of random distributed Ag adatoms on graphene sheet. The impurity concentration $n_{i}$ is $0.25 \%, 0.50 \%$, and $0.75 \%$, respectively (from left to right). The lattice simulation size is $2000 \times 2000$; the inset is a schematic image showing one silver atom connected to one carbon atom of a graphene sheet; $a=0.142 \mathrm{~nm}$ is the carbon-carbon bond length used for present calculations. (b) Real-space mapping of local density of states (LDOS) in Ag-adsorbed graphene $\left(n_{i}=\right.$ $0.30 \%$ ) at $E_{F}=0$; the unit of amplitude is $10^{17} \mathrm{eV}^{-1} \mathrm{~m}^{-2}$; the black dots denote the locations of Ag adatoms. (c) Relationship between LDOS and energy $E$ ( $E_{D}$ is the energy at the Dirac point) at four different locations marked by green symbols in (b); the inset is the change in LDOS in the region spanned by the black arrow in (b). (d) Relationship between average density of states (ADOS) and energy $E$ for Ag-adsorbed graphene with $n_{i}=0 \%, 0.1 \%, 0.3 \%, 0.5 \%, 1 \%$, and $5 \%$.

other areas. Figure 1(c) shows the curves of LDOS versus energy $E$ at four chosen places denoted by green symbols in Fig. 1(b). Obvious resonances emerge near the Dirac point; however, the intensity of these resonances does not decay monotonically with the distance from Ag impurities. The inset of Fig. 1(c) demonstrates the change of LDOS in the region spanned by the black arrow in Fig. 1(b) (between two $\mathrm{Ag}$ impurities). As we move from the Ag impurity on the left to the one on the right, LDOS fluctuates dramatically and many peaks appear in the middle. This is due to the multiple scattering of electronic waves from a large number of $\mathrm{Ag}$ adatoms, a phenomenon that could not be observed in single-impurity systems. The behavior of ADOS should better reflect the statistical properties of plenty of resonant impurities in graphene than the behavior of LDOS. Figure 1(d) shows the resonant peak of ADOS goes up as $n_{i}$ increases. Although scanning tunneling spectroscopy has proven to be a very effective method to locally probe resonant impurities [12-14,16], ADOS in graphene containing resonant impurities is still unexplored experimentally.

To experimentally detect the global behavior of $\mathrm{Ag}$ adatom-induced resonant impurities, we carried out quantum capacitance (QC) measurement of Ag-adsorbed graphene. The areas of our graphene samples and top-gate electrodes 
(a)
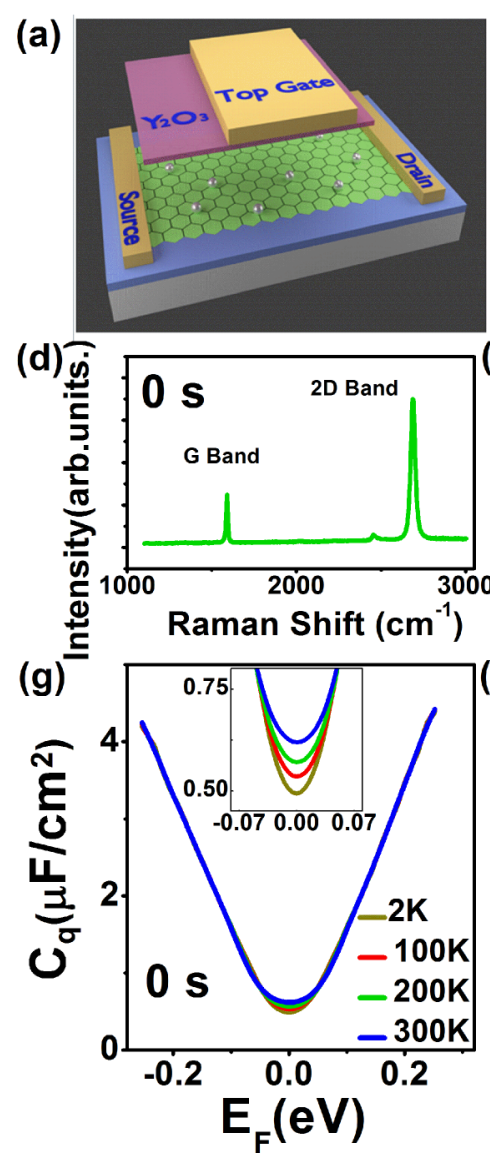

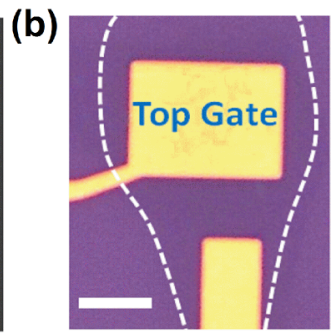

(e)
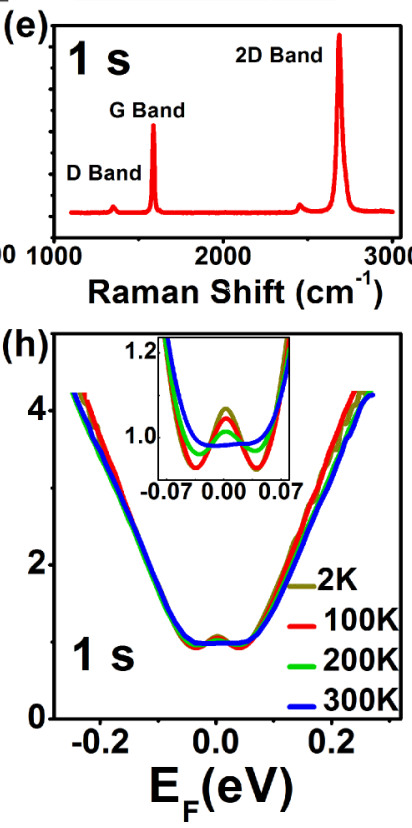
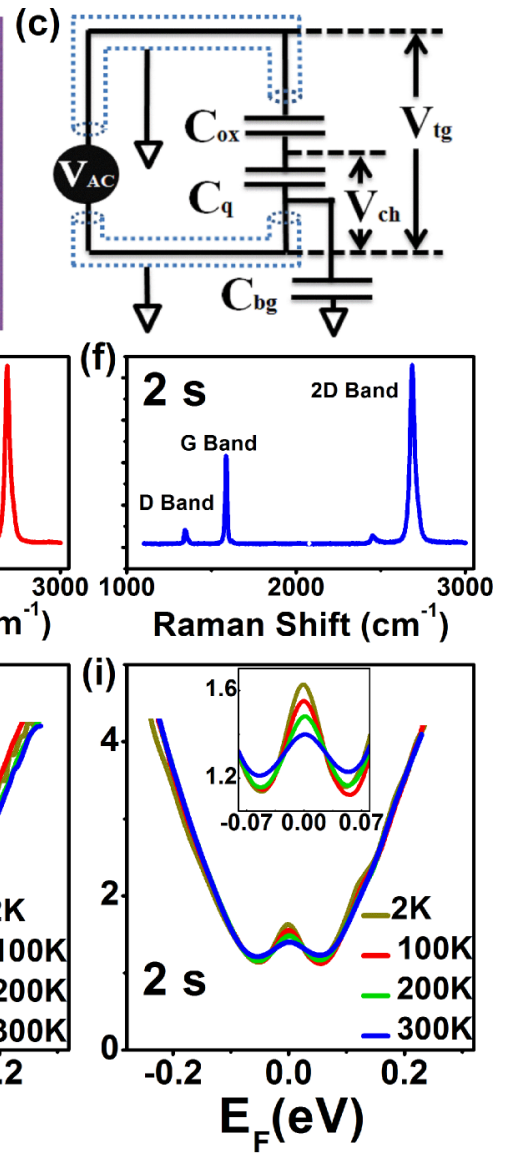

FIG. 2. (Color online) Experimental measurements for Ag-adsorbed graphene. (a) Schematics of a Ag-adsorbed graphene quantum capacitance device. (b) An optical image of our Ag-adsorbed graphene quantum capacitance device. Graphene flake is denoted by white dashed line, and the scale bar is $5 \mu \mathrm{m}$. (c) The setup for our quantum capacitance measurement. (d)-(f) Raman spectra for Samples A, B, and C, respectively. (g)-(i) The relationships between quantum capacitance $C_{q}$ and Fermi energy $E_{F}$ measured at different temperatures for Samples A, B, and C, respectively. The insets enlarge the area near the charge neutrality point.

are in the range of 50-100 $\mu \mathrm{m}^{2}$. Figures 2(a)-2(c) illustrate the Ag-adsorbed graphene capacitor and the circuit used for capacitance measurement [36-38,46]. Before the deposition of a $\mathrm{Y}_{2} \mathrm{O}_{3}$ insulating layer, $\mathrm{Ag}$ adatoms were first deposited on graphene (labeled as Samples A, B, and C) in high base vacuum conditions $\left(\sim 10^{-7}\right.$ torr $)$ for 0,1 , and $2 \mathrm{~s}$. Long deposition duration tends to introduce more Ag clusters rather than more Ag atoms; deposition durations of $2 \mathrm{~s}$ or less were chosen in this study [41].

Raman spectra in Figs. 2(d)-2(f) show that Ag adatoms cause slight structural changes of graphene. In contrast to the absence of a D-band for Sample A, a weak D-band peak related to lattice defects appears around $1345 \mathrm{~cm}^{-1}$ for Samples B and $C[1,34,37,47]$. The ratio of the intensity of $D$ band to $G$ band (i.e. $I_{\mathrm{D}} / I_{\mathrm{G}}$ ) increases by increasing deposition duration. Therefore, as Ag concentration increases, the honeycomb symmetric structure of graphene should be broken gradually $[18,19,34,38,47,48]$. As Raman spectra confirmed, these Ag adatoms apparently introduce some short-range disorder to graphene, although it was impossible to determine the details.

Quantum capacitance measurements in graphene top-gated devices whose insulating layer has a large oxide capacitance are very sensitive to the changes in ADOS if graphene contains resonant impurities [31,32]. Based on a serial connection model, quantum capacitance $C_{q}$ can be calculated by $C_{q}^{-1}=$ $C_{t g}^{-1}-C_{o x}^{-1}$, where $C_{t g}$ is the total measured top-gate capacitance, and $C_{o x}$ is the oxide capacitance of $\mathrm{Y}_{2} \mathrm{O}_{3}[31,32,36,37]$. The values of $C_{o x}$ were measured using a parallel-plate reference capacitor with an $\mathrm{Au} / \mathrm{Y}_{2} \mathrm{O}_{3} / \mathrm{Au}$ structure in which the $\mathrm{Y}_{2} \mathrm{O}_{3}$ layer was fabricated at the same time as that of graphene device. The ultra-thin $\mathrm{Y}_{2} \mathrm{O}_{3}$ layers are structurally uniform as characterized by cross-sectional high-resolution transmission electron microscopy, and their oxide capacitance increases with temperatures [41]. The data of $C_{t g}$ versus topgate voltage $V_{t g}$ are presented in Fig. S3 [41]. As the sample is cooling down, the overall reduction in $C_{t g}$ is a result of the decrease in temperature-dependent $C_{o x}$ of $\mathrm{Y}_{2} \mathrm{O}_{3}$ layer. The relationship between $C_{q}$ and $E_{F}$ can be calculated by $C_{q}^{-1}=$ $C_{t g}^{-1}-C_{o x}^{-1}$ and $E_{F}=e \int_{0}^{V_{t g}}\left(1-\frac{C_{t g}}{C_{o x}}\right) d V_{t g}$, where $e$ is the elementary charge [36,37]. For Sample A [Fig. 2(g)], the linear relationship between $C_{q}$ and $E_{F}$ is a typical characteristic of high-quality pristine graphene $[3,4,31-33,36,37,49,50]$. Our investigation of pristine graphene (Sample A) is necessary for a good understanding of the initial status of graphene samples before $\mathrm{Ag}$ deposition. The nonzero value of $C_{q}$ near the charge neutrality point (CNP) provides direct evidence for the existence of the residual carrier density resulting from 

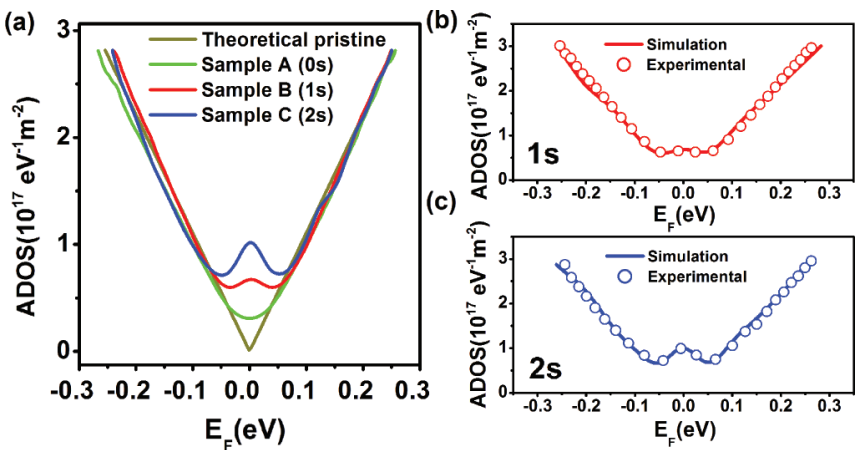

FIG. 3. (Color online) The ADOS results for Ag-adsorbed graphene fitted with the theoretical model. (a) Relationship between ADOS and Fermi energy $E_{F}$ measured at $T=2 \mathrm{~K}$ for Samples A, $\mathrm{B}$, and $\mathrm{C}$ (green, red, blue) and the theoretical results for perfect graphene for comparison (dark yellow). (b) and (c) The measured (circles) and calculated (solid line) relationships between ADOS and Fermi energy $E_{F}$ for Samples B and C, respectively.

inevitable ripples and charged impurities in real graphene devices [6,9,50-52]. For Samples B and C, the resonances near CNP [see Figs. 2(h) and 2(i)] directly confirm that Ag adatoms on graphene sheets behave as resonant impurities. However, it is difficult to identify Ag-induced resonant impurities through transport measurement [41]. A high concentration of impurities results in strong resonances as reflected by QC. Furthermore, the resonances obtained from QC look sensitive to temperature. Although DOS in the resonant state model should be temperature independent, the experimental observation that the resonant peak of $C_{q}$ near the CNP becomes intense with decreasing temperature can be well explained by considering the Fermi-Dirac distribution of electrons [41,31,53-56]. Thermal activation effect averages out these notable resonant features near CNP $[31,33,50]$.

To exclude thermal effects, we quantitatively studied the samples at $T=2 \mathrm{~K}$. Average DOS can be determined by $D=C_{q} / e^{2}$. The resulting curves of ADOS versus $E_{F}$ are shown in Fig. 3(a). The appearance of midgap states in disordered graphene's ADOS, although predicted by a large number of theoretical studies [5-10,17,21,25,42,57], has never been clearly demonstrated by other experimental methods. Quantum capacitance measurement of graphene devices can be used to study the overall effect of resonant impurities with different impurity concentrations $n_{i}$ on a large scale. The effective response of the Dirac resonance to impurity concentration is demonstrated here. Away from CNP, the unchanged linear slope after $\mathrm{Ag}$ deposition is consistent with our calculations, which is different from the behavior of Anderson type of disorder [46,58,59]. As shown in Figs. 3(b) and 3(c), the results of ADOS from tight-binding calculations are an exact replica of the experimental observations, leaving $n_{i}$ the only fitting parameter to be calculated $(0.30 \pm 0.01 \%$ for Sample B and $0.50 \pm 0.01 \%$ for Sample C). Our experimental data were also fitted with the model considering the density of resonant states characterized by the Thomas-Porter distribution [41-43].

Magnetocapacitance measurements of Sample B (1 s) at $T=2 \mathrm{~K}$ accompanied by tight-binding theoretical calculations reveal that $\mathrm{Ag}$ adatoms, as resonant impurities, influence
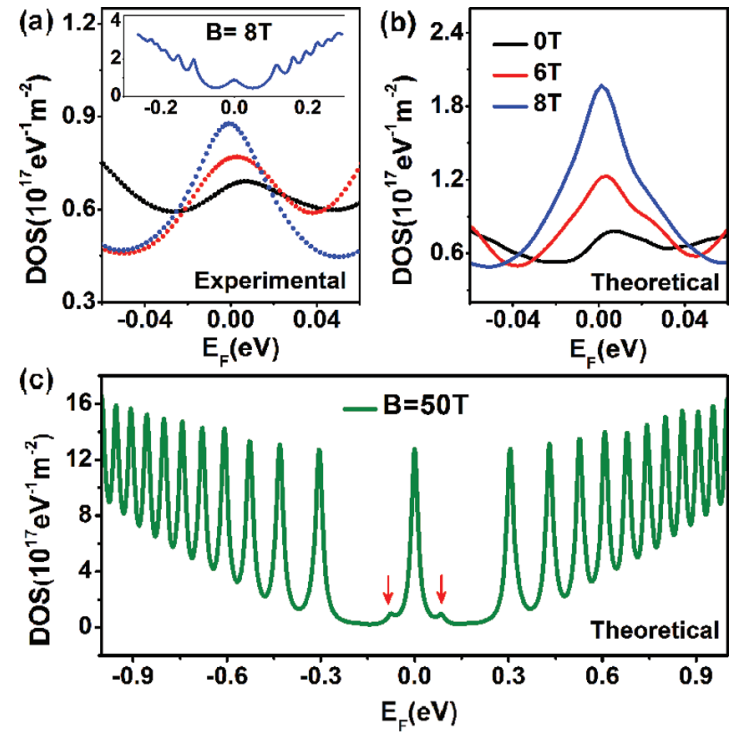

FIG. 4. (Color online) Magnetoquantum capacitance and ADOS of Ag-adsorbed graphene. (a) and (b) The experimental (dots) and tight-binding simulation (lines) results for the zero Landau level of Sample B at $B=0 \mathrm{~T}$ (black), $6 \mathrm{~T}$ (red), and $8 \mathrm{~T}$ (blue); the inset of (a) contains experimental results measured at $B=8 \mathrm{~T}$ and $T=2 \mathrm{~K}$ including high-energy Landau levels. (c) Tight-binding calculation results for Ag-adsorbed graphene at $B=50 \mathrm{~T}$. The sample size for theoretical calculation is $3200 \times 3200$ carbon atoms. The on-site energy $\left(\varepsilon_{d}=0\right)$, energy-dependent effective potential $(V=0.08 t)$, and impurity concentration $\left(n_{i}=0.30 \%\right)$ are extracted from previous theoretical simulations.

Landau-level quantization of graphene. As shown in the inset of Fig. 4(a), high-energy Landau levels oscillate remarkably in a magnetic field of strength $B=8 \mathrm{~T}$, indicating that the $\mathrm{Ag}$ resonant impurities serve as mild disorder and do not broaden Landau levels seriously $[6,8,17,33,57,60]$. More interestingly, as $B$ increases, the zeroth Landau level gradually starts to appear and play a dominant role; therefore, the central peak (a combination of resonant peak and the zeroth Landau-level peak) gradually moves away from the resonant peak measured under $B=0 \mathrm{~T}$ [see Fig. 4(a)]. In comparison with the central peak measured under $B=8 \mathrm{~T}$, there is a relative shift about $6 \mathrm{~m} \mathrm{eV}$ for resonant states. Even though density functional theory simulations yield the resonance energy $E_{i}=$ 0 for graphene adsorbed with $\mathrm{Ag}$ adatoms, the experimental observation of $E_{i}=+6 \mathrm{meV}$ is still reasonable due to the doping effect inevitably introduced during the device fabrication process. The tight-binding calculations with $E_{i}=$ $+6 \mathrm{meV}$ theoretically verified the left shift of the central peak as $B$ increases [Fig. 4(b)]. Another important feature of Landau-level quantization influenced by resonant impurities is the splitting of the central peak, as shown by tight-binding calculation results [Figs. 4(b) and 4(c)] $[6,8,57,60]$. Two subbands grow from the zeroth Landau-level peak due to the hybridization effect of $\mathrm{Ag}$ adatoms and graphene. Both of them move away from CNP with increasing $B$, but the experimental observation with $B$ up to $8 \mathrm{~T}$ is not able to clearly show these subbands as this feature could have been easily smeared by small amounts of charged impurities and electron-hole puddles 
[Fig. 4(a)] [20,33,53-56]. The fluctuation of carrier density is evidenced by the smearing feature at CNP and the broadening effect of Landau levels. With a large enough magnetic field (e.g. $B=50 \mathrm{~T}$ ), it should be possible to observe the splitting feature clearly, as our tight-binding calculations suggest.

Quantum capacitance measurement is a powerful method to study the electronic behavior of the system containing resonant impurities. This simple but effective method has obvious advantages in studying the global behavior of various disorders in graphene on a large scale. The investigation of interaction effects among electrons and resonant impurities or the multiple scattering between different types of resonant impurities can be further realized experimentally using QC methods. In fact, electron-electron interactions in graphene have been well investigated by QC measurements [27,61-63]. Resonant impurities significantly modify the band structure of graphene with the emergence of dispersionless bands. By combining this exotic band dispersion with graphene's extraordinary properties, more interesting physical phenomena are expected to come out.

\section{CONCLUSION}

We demonstrated the direct experimental observation of midgap states in graphene realized by QC measurements. The Ag-adatom-induced resonant peak as measured by quantum capacitance is mainly dependent on Ag-adatom concentrations and magnetic fields, in good agreement with theoretical calculations based on density functional theory and tightbinding methods. The resonant peak in ADOS and the splitting of the zeroth Landau-level peak for Ag-adsorbed graphene are manifestations of the formation of flat impurity bands near the Dirac point, resulting in the resonant scattering near the Dirac point but without significant changes far away from the Dirac point. Ag adatoms induce midgap states but with minimal structural changes and very weak localization effects, as evidenced by our systematic studies including theoretical calculations, Raman scattering, capacitance, and transport measurements. The modification of electronic properties by $\mathrm{Ag}$ adatoms occurring only at the Dirac point is useful for designing new device structures particularly involving band-structure engineering.

\section{ACKNOWLEDGMENTS}

The authors are grateful for the helpful discussion from P. Sheng, Z. Q. Zhang, K. T. Law, and H. B. Chan of Department of Physics, HKUST, and D. N. Sheng of Department of Physics and Astronomy, California State University, and Dr. Z. F. Wang of Department of Physics, University of Utah. Financial support from the Research Grants Council of Hong Kong (Project Nos. 604112, N_HKUST613/12 and HKUST9/CRF/08) and technical support of the Raith-HKUST Nanotechnology Laboratory for the electron-beam lithography facility (Project No. SEG HKUST08) are hereby acknowledged.
[1] K. S. Novoselov, A. K. Geim, S. V. Morozov, D. Jiang, Y. Zhang, S. V. Dubonos, I. V. Grigorieva, and A. A. Firsov, Science 306, 666 (2004).

[2] P. M. Ostrovsky, I. V. Gornyi, and A. D. Mirlin, Phys. Rev. B 74, 235443 (2006).

[3] A. H. Castro Neto, F. Guinea, N. M. R. Peres, K. S. Novoselov, and A. K. Geim, Rev. Mod. Phys. 81, 109 (2009).

[4] N. M. R. Peres, Rev. Mod. Phys. 82, 2673 (2010).

[5] V. M. Pereira, F. Guinea, J. M. B. L. Dos Santos, N. M. R. Peres, and A. H. C. Neto, Phys. Rev. Lett. 96, 036801 (2006).

[6] T. O. Wehling, M. I. Katsnelson, and A. I. Lichtenstein, Chem. Phys. Lett. 476, 125 (2009).

[7] T. O. Wehling, M. I. Katsnelson, and A. I. Lichtenstein, Phys. Rev. B 80, 085428 (2009).

[8] S. J. Yuan, H. De Raedt, and M. I. Katsnelson, Phys. Rev. B 82, 115448 (2010).

[9] J. P. Robinson, H. Schomerus, L. Oroszlány, and V. I. Fal'ko, Phys. Rev. Lett. 101, 196803 (2008).

[10] T. Stauber, N. M. R. Peres, and F. Guinea, Phys. Rev. B 76, 205423 (2007).

[11] G. Kresse and J. Hafner, J. Phys.: Condens. Matter 6, 8245 (1994).

[12] M. M. Ugeda, I. Brihuega, F. Guinea, and J. M. GomezRodriguez, Phys. Rev. Lett. 104, 096804 (2010).

[13] M. M. Ugeda, D. Fernández-Torre, I. Brihuega, P. Pou, A. J. Martínez-Galera, R. Pérez, and J. M. Gómez-Rodríguez, Phys. Rev. Lett. 107, 116803 (2011).
[14] V. W. Brar, R. Decker, H.-M. Solowan, Y. Wang, L. Maserati, K. T. Chan, H. Lee, C. O. Girit, A. Zettl, S. G. Louie, M. L. Cohen, and M. F. Crommie, Nat. Phys. 7, 43 (2011).

[15] A. Saffarzadeh and G. Kirczenow, Phys. Rev. B 85, 245429 (2012).

[16] M. Scheffler, D. Haberer, L. Petaccia, M. Farjam, R. Schlegel, D. Baumann, T. Hänke, A. Grüneis, M. Knupfer, C. Hess, and B. Büchner, Acs Nano 6, 10590 (2012).

[17] T. O. Wehling, A. V. Balatsky, M. I. Katsnelson, A. I. Lichtenstein, K. Scharnberg, and R. Wiesendanger, Phys. Rev. B 75, 125425 (2007).

[18] J. H. Chen, W. G. Cullen, C. Jang, M. S. Fuhrer, and E. D. Williams, Phys. Rev. Lett. 102, 236805 (2009).

[19] Z. H. Ni, L. A. Ponomarenko, R. R. Nair, R. Yang, S. Anissimova, I. V. Grigorieva, F. Schedin, P. Blake, Z. W. Shen, E. H. Hill, K. S. Novoselov, and A. K. Geim, Nano Lett. 10, 3868 (2010).

[20] J. H. Chen, C. Jang, S. Adam, M. S. Fuhrer, E. D. Williams, and M. Ishigami, Nat. Phys. 4, 377 (2008).

[21] T. O. Wehling, S. Yuan, A. I. Lichtenstein, A. K. Geim, and M. I. Katsnelson, Phys. Rev. Lett. 105, 056802 (2010).

[22] A. Ferreira, J. Viana-Gomes, J. Nilsson, E. R. Mucciolo, N. M. R. Peres, and A. H. Castro Neto, Phys. Rev. B 83, 165402 (2011).

[23] D. C. Elias, R. R. Nair, T. M. G. Mohiuddin, S. V. Morozov, P. Blake, M. P. Halsall, A. C. Ferrari, D. W. Boukhvalov, M. I. Katsnelson, A. K. Geim, and K. S. Novoselov, Science 323, 610 (2009). 
[24] C. Bena, Phys. Rev. Lett. 100, 076601 (2008).

[25] S. D. Wu, L. Jing, Q. X. Li, Q. W. Shi, J. Chen, H. B. Su, X. P. Wang, and J. L. Yang, Phys. Rev. B 77, 195411 (2008).

[26] J.-H. Chen, L. Li, W. G. Cullen, E. D. Williams, and M. S. Fuhrer, Nat. Phys. 7, 535 (2011).

[27] L. Wang, Y. Wang, X. L. Chen, W. Zhu, C. Zhu, Z. F. Wu, Y. Han, M. W. Zhang, W. Li, Y. H. He, K. T. Law, D. S. Su, and N. Wang, Sci. Rep. 3, 2041 (2013).

[28] K. T. Chan, J. B. Neaton, and M. L. Cohen, Phys. Rev. B 77, 235430 (2008).

[29] X. J. Liu, C. Z. Wang, Y. X. Yao, W. C. Lu, M. Hupalo, M. C. Tringides, and K. M. Ho, Phys. Rev. B 83, 235411 (2011).

[30] S. Luryi, Appl. Phys. Lett. 52, 501 (1988).

[31] S. Droscher, P. Roulleau, F. Molitor, P. Studerus, C. Stampfer, K. Ensslin, and T. Ihn, Appl. Phys. Lett. 96, 152104 (2010).

[32] J. L. Xia, F. Chen, J. H. Li, and N. J. Tao, Nature Nanotechnology 4, 505 (2009).

[33] L. A. Ponomarenko, R. Yang, R. V. Gorbachev, P. Blake, A. S. Mayorov, K. S. Novoselov, M. I. Katsnelson, and A. K. Geim, Phys. Rev. Lett. 105, 136801 (2010).

[34] A. C. Ferrari, J. C. Meyer, V. Scardaci, C. Casiraghi, M. Lazzeri, F. Mauri, S. Piscanec, D. Jiang, K. S. Novoselov, S. Roth, and A. K. Geim, Phys. Rev. Lett. 97, 187401 (2006).

[35] Z. X. Wang, H. Xu, Z. Zhang, S. Wang, L. Ding, Q. Zeng, L. Yang, T. Pei, X. Liang, M. Gao, and L.-M. Peng, Nano Lett. 10, 2024 (2010).

[36] H. L. Xu, Z. Y. Zhang, Z. X. Wang, S. Wang, X. L. Hang, and L. M. Peng, Acs Nano 5, 2340 (2011).

[37] L. Wang, X. L. Chen, Y. Wang, Z. F. Wu, W. Li, Y. Han, M. W. Zhang, Y. H. He, C. Zhu, K. K. Fung, and N. Wang, Nanoscale 5, 1116 (2013).

[38] W. Li, Y. H. He, L. Wang, G. H. Ding, Z. Q. Zhang, R. W. Lortz, P. Sheng, and N. Wang, Phys. Rev. B 84, 045431 (2011).

[39] Y. H. He, L. Wang, X. L. Chen, Z. F. Wu, W. Li, Y. Cai, and N. Wang, Appl. Phys. Lett. 99, 033109 (2011).

[40] A. Hazeghi, J. A. Sulpizio, G. Diankov, D. Goldhaber-Gordon, and H. S. P. Wong, Rev. Sci. Instrum. 82, 5 (2011).

[41] See Supplemental Material at http://link.aps.org/supplemental/ 10.1103/PhysRevB.89.075410 for the details of theoretical simulation of single Ag adatom absorbed on single layer graphene, the details of the capacitance data of graphene decorated with $\mathrm{Ag}$ clusters, the details of structure and property of $\mathrm{Y}_{2} \mathrm{O}_{3}$ dielectric layers, the details of transport properties of $\mathrm{Ag}$ adsorbed single layer graphene, the details of the simulation results of temperature dependent quantum capacitance by considering the Fermi Dirac distribution, and the details of the fitting experiemental data with a theoretical model considering the density of resonant states characterized by the Thomas Porter distribution.

[42] B. L. Huang and C. Y. Mou, Europhys. Lett. 88, 6 (2009).

[43] B.-L. Huang, M.-C. Chang, and C.-Y. Mou, Phys. Rev. B 82, 155462 (2010).

[44] W. Zhu, W. Li, Q. W. Shi, X. R. Wang, X. P. Wang, J. L. Yang, and J. G. Hou, Phys. Rev. B 85, 073407 (2012).

[45] A. P. Jauho and J. W. Wilkins, Phys. Rev. B 28, 4628 (1983).

[46] W. Li, X. L. Chen, L. Wang, Y. H. He, Z. F. Wu, Y. Cai, M. W. Zhang, Y. Wang, Y. Han, R. W. Lortz, Z. Q. Zhang, P. Sheng, and N. Wang, Sci. Rep. 3, 1772 (2013).

[47] A. C. Ferrari, Solid State Commun. 143, 47 (2007).

[48] L. G. Cancado et al., Appl. Phys. Lett. 88, 3106 (2006).

[49] K. S. Novoselov, A. K. Geim, S. V. Morozov, D. Jiang, M. I. Katsnelson, I. V. Grigorieva, S. V. Dubonos, and A. A. Firsov, Nature 438, 197 (2005).

[50] B. Y. K. Hu, E. H. Hwang, and S. Sarma, Phys. Rev. B 78 , 165411 (2008).

[51] A. Fasolino, J. H. Los, and M. I. Katsnelson, Nat. Mater. 6, 858 (2007).

[52] S. Adam, E. H. Hwang, V. M. Galitski, and S. Das Sarma, Proc. Nat. Acad. Sci. USA 104, 18392 (2007).

[53] J. Martin, N. Akerman, G. Ulbricht, T. Lohmann, J. H. Smet, K. Von Klitzing, and A. Yacoby, Nat. Phys. 4, 144 (2008).

[54] H. L. Xu, Z. Y. Zhang, and L. M. Peng, Appl. Phys. Lett. 98 , $133122(2011)$.

[55] Q. Z. Li, E. H. Hwang, and S. Das Sarma, Phys. Rev. B 84, 115442 (2011).

[56] M. Gibertini, A. Tomadin, F. Guinea, M. I. Katsnelson, and M. Polini, Phys. Rev. B 85, 201405 (2012).

[57] V. M. Pereira, J. dos Santos, and A. H. Castro, Phys. Rev. B 77, 115109 (2008)

[58] S. J. Xiong and Y. Xiong, Phys. Rev. B 76, 214204 (2007).

[59] B. Dóra, K. Ziegler, and P. Thalmeier, Phys. Rev. B 77, 115422 (2008).

[60] W. Zhu, Q. W. Shi, X. R. Wang, J. Chen, J. L. Yang, and J. G. Hou, Phys. Rev. Lett. 102, 056803 (2009).

[61] G. L. Yu, R. Jalil, B. Belle, A. S. Mayorov, P. Blake, R. Schedin, S. V. Morozov, L. A. Ponomarenko, F. Chiappini, S. Wiedmann, U. Zeitler, M. I. Katsnelson, A. K. Geim, K. S. Novoselov, and D. C. Elias, Proc. Nat. Acad. Sci. USA 110, 3282 (2013).

[62] X. L. Chen, L. Wang, W. Li, Y. Wang, Y. H. He, Z. F. Wu, Y. Han, M. W. Zhang, W. Xiong, and N. Wang, Appl. Phys. Lett. 102, 203103 (2013).

[63] X. L. Chen, L. Wang, W. Li, Y. Wang, Z. F. Wu, M. W. Zhang,Y. Han, Y. H. He, and N. Wang, Nano Research 6, 619 (2013). 